\title{
BIFURCATION IN NONLINEAR STURM-LIOUVILLE PROBLEMS WITH INDEFINITE WEIGHT AND SPECTRAL PARAMETER IN THE BOUNDARY CONDITION
}

\author{
ULKAR V. GURBANOVA
}

\begin{abstract}
In this paper, we consider the nonlinear Sturm-Liouville problem with an indefinite weight and a spectral parameter in the boundary condition. We establish the existence of four families of global solutions branches bifurcating from the points of the line of trivial solutions and possessing the usual oscillation properties.
\end{abstract}

\section{Introduction}

We consider the nonlinear Sturm-Liouville problem

$$
\begin{gathered}
\ell y \equiv-\left(p(x) y^{\prime}\right)^{\prime}+q(x) y=\lambda r(x) y+g\left(x, y, y^{\prime}, \lambda\right), x \in(0,1), \\
b_{0} y(0)=d_{0} p(0) y^{\prime}(0), \\
\left(a_{1} \lambda+b_{1}\right) y(1)=p(1) y^{\prime}(1),
\end{gathered}
$$

where

(i) $p \in C^{1}[0,1], q, r \in C[0,1], p>0, q \geq 0$ and $r$ changes sign on $[0,1]$,

(ii) $b_{0}, d_{0}, a_{1}, b_{1}$ are real constants such that

$\left|b_{0}\right|+\left|d_{0}\right|>0, b_{0} d_{0} \geq 0$ and, if $b_{0}=0$, then $q \not \equiv 0$, and $a_{1}>0, b_{1} \leq 0$.

(iii) $g \in C\left([0,1] \times \mathbb{R}^{3}\right)$ and satisfy the following conditions:

$$
\begin{gathered}
u g(x, u, s, 0) \leq 0,(x, u, s) \in[0,1] \times \mathbb{R}^{2} ; \\
g(x, u, s, \lambda)=o(|u|+|s|) \text { as }|u|+|s| \rightarrow 0,
\end{gathered}
$$

uniformly in $(x, \lambda) \in[0,1] \times \Lambda$, for any bounded interval $\Lambda \subset \mathbb{R}$.

Problems of the form (1.1)-(1.3) arise in the study of various problems of mechanics, physics and biology; for example, problem (1.1)-(1.3) with $a_{1}=0$ arise from a selection-migration model in population genetics (see $[10,12])$.

Bifurcation of solutions of nonlinear Sturm-Liouville problems with a definite weight function was studied in $[2,5,8,17-19]$. These papers prove the existence of unbounded global continua of nontrivial solutions that having fixed oscillation count and emanating from bifurcation points and intervals surrounding the eigenvalues of the corresponding linear problems. Similar results for nonlinear

2010 Mathematics Subject Classification. 34B09, 34B15, 34C10, 34C23, 34K11, 34L20, 34K18, 47J10, 47J15.

Key words and phrases. Sturm-Liouville problem, indefinite weight, spectral parameter in the boundary condition, bifurcation point, oscillation count, global bifurcation. 
Sturm-Liouville problems with definite weight functions and a spectral parameter in the boundary conditions have been obtained in $[2,6]$. In the case when the weight function changes sign the bifurcation of solutions of nonlinear SturmLiouville problems have been studied in recent works $[3,4,16]$ in which shows the existence of four families of such global continua.

In this paper, we study behavior of global continua of solutions of problem (1.1)-(1.3) bifurcating from all trivial solutions corresponding to the eigenvalues of the linear problem obtained from (1.1)-(1.3) by setting nonlinear term $g$ to zero.

\section{Preliminary}

We consider the linear Sturm-Liouville problem

$$
\begin{aligned}
& \left(p(x) y^{\prime}(x)\right)^{\prime}+q(x) y(x)=\lambda r(x) y(x), x \in(0,1), \\
& b_{0} y(0)=d_{0} p(0) y^{\prime}(0),\left(a_{1} \lambda+b_{1}\right) y(1)=p(1) y^{\prime}(1) .
\end{aligned}
$$

Remark 2.1. Theorem 3.2 of [7] implies that the spectral problem (2.1) has a two infinite sequence of real and simple eigenvalues $\lambda_{n \pm}, n=1,2, \ldots$ satisfying

$$
0<\lambda_{1+}<\lambda_{2+}<\ldots<\lambda_{n+}<\ldots
$$

and

$$
0>\lambda_{1-}>\lambda_{2-}>\ldots>\lambda_{n-}>\ldots
$$

Moreover, the eigenfunction $y_{n \pm}(x), n \in \mathbb{N}$, corresponding to the eigenvalue $\lambda_{n}$ have exactly $n-1$ simple zeros in $(0,1)$.

It is well known that problem (2.1) reduces to the eigenvalue problem for a pair of linear operators $A: D(A) \subset H \rightarrow H$ and $R: H \rightarrow H$, where $H=L_{2}(0,1) \oplus \mathbb{C}$ is a Hilbert space with inner product

$$
\begin{gathered}
(\hat{y}, \hat{v})=(\{y, \alpha\},\{v, \beta\})=\int_{0}^{1} y(x) \overline{v(x)} d x+a_{1}^{-1} \alpha \bar{\beta}, \\
D(A)=\left\{\hat{y}=\{y, \alpha\} \in H: y, p y^{\prime} \in A C[0,1], \ell(y) \in L_{2}(0,1),\right. \\
\left.b_{0} y(0)=d_{0} p(0) y^{\prime}(0), \alpha=a_{1} y(1)\right\}, \\
A \hat{y}=A\{y, \alpha\}=\left\{\ell(y), p(1) y^{\prime}(1)-b_{1} y(1)\right\},
\end{gathered}
$$

and

$$
R \hat{y}=R\{y, \alpha\}=\{r y, \alpha\} .
$$

Therefore, problem (1.1)-(1.3) is equivalent to the following spectral problem

$$
A \hat{y}=\lambda R \hat{y}, \hat{y} \in D(A),
$$

i.e., the eigenvalues $\lambda_{n, \pm}, n \in \mathbb{N}$, of problem (2.1) and the operator $A$ coincide, and between the eigenvectors, there is a one-to-one correspondence

$$
y_{n, \pm} \leftrightarrow \hat{y}_{n, \pm}=\left\{y_{n, \pm}, \alpha_{n, \pm}\right\}, \alpha_{n, \pm}=a_{1} y_{n, \pm}(1) .
$$

Since $a_{1}>0$ it follows from $[13,20]$ that $A$ is a self-adjoint operator on $D(A)$.

Lemma 2.1. The operator $A$ is definite positive on $D(A)$. 
Proof. In view of (1.4), by (2.2) for any $\hat{y} \in D(A)$ we have

$$
\begin{gathered}
(A \hat{y}, \hat{y})=\int_{0}^{1} \ell(y)(x) \overline{y(x)} d x+a_{1}^{-1}\left(p(1) y^{\prime}(1)-b_{1} y(1)\right) a_{1} \overline{y(1)}= \\
\int_{0}^{1}\left\{p(x)\left|y^{\prime}(x)\right|^{2}+q(x)|y(x)|^{2}\right\} d x-p(1) y^{\prime}(1) \overline{y(1)}+p(0) y^{\prime}(0) \overline{y(0)}+ \\
p(1) y^{\prime}(1) \overline{y(1)}-b_{1}|y(1)|^{2}=\int_{0}^{1}\left\{p(x)\left|y^{\prime}(x)\right|^{2}+q(x)|y(x)|^{2}\right\} d x+ \\
N[y]-b_{1}|y(1)|^{2}>0
\end{gathered}
$$

where

$$
N[y]=\left(b_{0} / d_{0}\right) y^{2}(0) \geq 0 \text { for } d_{0} \neq 0, \quad N[y]=0 \text { for } d_{0}=0 .
$$

The proof of this lemma is complete.

Along with problem (2.3) we consider the following eigenvalue problem

$$
A \hat{y}-\lambda R \hat{y}=\mu \hat{y}, \hat{y} \in D(A),
$$

which is equivalent to the regular Sturm-Liouville problem with a spectral parameter in boundary condition:

$$
\begin{gathered}
\left(p(x) y^{\prime}(x)\right)^{\prime}+q(x) y(x)-\lambda r(x) y(x)=\mu y(x), x \in(0,1), \\
b_{0} y(0)=d_{0} p(0) y^{\prime}(0),\left(a_{1} \lambda+b_{1}\right) y(1)=p(1) y^{\prime}(1) .
\end{gathered}
$$

It follows from [13] that for each $\lambda \in \mathbb{R}$ the eigenvalues of (2.6) (or (2.5)) are real and simple, and forms an unboundedly increasing sequence $\left\{\mu_{n}(\lambda)\right\}_{n=1}^{\infty}$. Moreover, the eigenfunction $y_{n}(x, \lambda)$ corresponding to $\mu_{n}(\lambda)$ has $n-1$ simple zeros in $(0,1)$.

Remark 2.2. The number $\lambda$ is an eigenvalue of (1.1)-(1.3) if and if $\mu_{n}(\lambda)=0$.

We introduce the following notations:

$$
\begin{gathered}
b c_{0}=\left\{y \in C^{1}[0,1]: b_{0} y(0)=d_{0} y^{\prime}(0)\right\}, \\
b \hat{c}_{0}=\left\{\hat{y}: y \in b c_{0}\right\}, \\
b c_{1}^{\lambda}=\left\{y \in C^{1}[0,1]:\left(a_{1} \lambda+b_{1}\right) y(1)=p(1) y^{\prime}(1)\right\} \text { for each } \lambda \in \mathbb{R} .
\end{gathered}
$$

As is known (see $[11,14]$ ) that the $n$-th eigenvalue of $(2.5)$ can be characterized as:

$$
\mu_{n}(\lambda)=\max _{\hat{V}_{n-1}} \min _{\hat{y} \in b \hat{c}_{0}}\left\{\frac{(A \hat{y}, \hat{y})-\lambda(R \hat{y}, \hat{y})}{(\hat{y}, \hat{y})}:(\hat{y}, \hat{v})=0, \hat{v} \in \hat{V}_{n-1}\right\},
$$

where $\hat{V}_{n-1}$ is any set of linearly independent functions $\hat{v}_{j} \in b \hat{c}_{0}, j=, 2, \ldots n$. Then it follows that

$$
\mu_{n}(\lambda)=\max _{V_{k-1}} \min _{y \in b c_{0}}\left\{\mathcal{R}(y): \int_{0}^{1} y(x) \nu(x) d x+y(1) \nu(1)=0, v \in V_{n-1}\right\}
$$

where

$$
\mathcal{R}(y)=\frac{\int_{0}^{1}\left\{p(x) y^{\prime 2}(x)+q(x) y^{2}(x)\right\} d x-\lambda \int_{0}^{1} r(x) y^{2}(x) d x+N[y]}{\int_{0}^{1} y^{2}(x) d x+b_{1} y^{2}(1)},
$$


and

$$
V_{n-1}=\left\{y: \hat{y} \in \hat{V}_{n-1}\right\} .
$$

Let $(\lambda, y)$ be a solution of (2.1). Then multiplying both sides of (1.1) by $y(x)$, integrating this result from 0 to 1 , and using boundary conditions (1.2) and (1.3) we obtain

$$
\int_{0}^{1}\left\{p(x) y^{\prime 2}(x)+q(x) y^{2}(x)\right\} d x+N[y]=\lambda\left\{\int_{0}^{1} r(x) y^{2}(x) d x+a_{1} y^{2}(1) .\right\}
$$

By (1.4) it follows from (2.8) that

$$
\begin{aligned}
& \int_{0}^{1} r(x) y^{2}(x) d x+a_{1} y^{2}(1)>0 \text { if } \lambda>0, \\
& \int_{0}^{1} r(x) y^{2}(x) d x+a_{1} y^{2}(1)<0 \text { if } \lambda<0 .
\end{aligned}
$$

\section{Classes of usual oscillation count and reducing problem}

\section{(1.1)-(1.3) to the equivalent operator equation}

Let $E$ be the Banach space $E=C^{1}[0,1] \cap b c_{0}$ with the usual norm

$$
\|y\|_{1}=\max _{x \in[0,1]}|y(x)|+\max _{x \in[0,1]}\left|y^{\prime}(x)\right|,
$$

and let $\hat{E}$ be the Banach space $\hat{E}=E \oplus \mathbb{C}$ with the norm

$$
\|\hat{y}\|_{1}=\|\{y, \alpha\}\|_{1}=\|y\|_{1}+|\alpha| .
$$

If $\{y, \alpha\} \in D(A)$, then $y^{\prime} \in A C[0,1]$ in view of $p \in C^{1}[0,1]$. Hence it follows that $y \in C^{1}[0,1]$ and $D(A) \subseteq \hat{E}$.

For each fixed $\lambda \in \mathbb{R}$ let by $S_{n, \lambda}^{\sigma, \nu}, n \in \mathbb{N}, \sigma \in\{+,-\}$ and $\nu \in\{+,-\}$ we denote the set of functions $y \in E$ that satisfy the following conditions:

(i) $y \in b c_{1}^{\lambda}$,

(ii) $y(x)$ has exactly $n-1$ simple zeros in $(0,1)$,

(iii) $\sigma \int_{0}^{1} r(x) y^{2}(x) d x+a_{1} y^{2}(1)>0$,

(iv) $\lim _{x \rightarrow 0+} \nu y(x)=1$.

Now for each $n \in \mathbb{N}$, each $\sigma \in\{+,-\}$ and each $\nu \in\{+,-\}$ let $S_{n, \lambda}^{\sigma, \nu}$ and $S_{n}^{\sigma, \nu}$ be sets are defined as follows:

$$
\begin{gathered}
S_{n}^{\sigma, \nu}=\bigcup_{\lambda \in R^{\sigma}} S_{n, \lambda}^{\sigma, \nu}, \\
S_{n}^{\sigma}=S_{n}^{\sigma,+} \cap S_{n}^{\sigma,-} .
\end{gathered}
$$

For each $n \in \mathbb{N}$ and each $\sigma \in\{+,-\}$ the sets $S_{n}^{\sigma,+}, S_{n}^{\sigma,-}$ and $S_{n}^{\sigma}$ are open subsets in $E$. Moreover, if $\hat{y} \in \partial S_{n}^{\sigma}$, then either

(i) there is a $\eta \in[0,1]$ such that $y(\eta)=y^{\prime}(\eta)=0$, or

(ii) $\int_{0}^{1} r(x) y^{2}(x) d x+a_{1} y^{2}(1)=0$.

Let now

$$
\hat{S}_{n}^{\sigma,+}=\left\{\hat{y} \in \hat{E}: y \in S_{n}^{\sigma,+}\right\}, \hat{S}_{n}^{\sigma,-}=\left\{\hat{y} \in \hat{E}: y \in S_{n}^{\sigma,-}\right\},
$$


and

$$
\hat{S}_{n}^{\sigma}=S_{n}^{\sigma,+} \cup S_{n}^{\sigma,-} .
$$

We define the continuous operators $R: \hat{E} \rightarrow C^{0}[0,1]$ and $G: \mathbb{R} \times \hat{E} \rightarrow$ $C^{0}[0,1] \oplus \mathbb{C}$ by

$$
R(\lambda, \hat{y})=R(\lambda,\{y, \alpha\})=\{r(x) y, \alpha\}, \alpha=a_{1} y(1)
$$

and

$$
G(\lambda, \hat{y})=G(\lambda,\{y, \alpha\})=\left\{g\left(x, y, y^{\prime}, \lambda\right), 0\right\},
$$

respectively, where $C^{0}[0,1] \oplus \mathbb{R}$ has norm given by

$$
\|\hat{y}\|_{0}=\|\{y, \alpha\}\|_{0}=\|y\|_{0}+|\alpha|,\|y\|_{0}=\max _{x \in[0,1]}|y(x)| .
$$

Then (1.1)-(1.3) is reduced to the following equivalent problem

$$
A \hat{y}=\lambda R \hat{y}+G(\lambda, \hat{y}),
$$

i.e., between the solutions $(\lambda, y)$ and $(\lambda, \hat{y})$ of problems (1.1)-(1.3) and (3.1) there is a one-to-one correspondence

$$
(\lambda, y) \leftrightarrow(\lambda, \hat{y}), \hat{y}=\{y, \alpha\}, \alpha=a_{1} y(1) .
$$

Since $\lambda=0$ is not eigenvalue of linear problem (2.1) it follows from [6, Lemma 3.3] that there exists

$$
\mathcal{A}=A^{-1}: C^{0}[0,1] \oplus \mathbb{C} \rightarrow D(A)
$$

and is a continuous and compact operator.

Let $\mathcal{R}: \hat{E} \rightarrow \hat{E}$ and $\mathcal{G}: \mathbb{R} \times \hat{E} \rightarrow \hat{E}$ be the operators defined by

$$
\mathcal{R}=\mathcal{A} R \text { and } \mathcal{G}=\mathcal{A} G,
$$

respectively. Then $\mathcal{R}$ and $\mathcal{G}$ are also continuous and compact operators. Moreover, it follows from (1.6) that

$$
\mathcal{G}(\lambda, \hat{y})=o\left(\|\hat{y}\|_{1}\right) \text { as }\|\hat{y}\|_{1} \rightarrow 0
$$

uniformly in $\lambda \in \Lambda$ (see $[2,4])$.

It is obvious that the nonlinear eigenvalue problem (1.1)-(1.3) (or (3.1)) can be rewritten in the following equivalent operator equation

$$
\hat{y}=\lambda \mathcal{R} \hat{y}+\mathcal{G}(\lambda, \hat{y})
$$

By (3.3) problem (3.4) is linearizable, and the linearization of this problem at $\hat{y}=\hat{0}=\{0,0\}$ is given by

$$
\hat{y}=\lambda \mathcal{R} \hat{y}
$$

Note that problem (3.5), in turn, is equivalent to problem (2.3) (or (2.1)). 
4. Global bifurcation from zero in problem (1.1)-(1.3)

The following results are needed in the sequel.

Lemma 4.1. If $(\lambda, y)$ is a nontrivial solution of (1.1)-(1.3) such that $y \in \partial S_{n}^{\sigma, \nu}$, then $y \equiv 0$.

The proof of Lemma 4.1 is similar to the proof of [1, Lemma 1.1].

Lemma 4.2. Let $(\lambda, y)$ is a nontrivial solution of (1.1)-(1.3). Then $\lambda \neq 0$.

Proof. Multiplying both sides of (1.1) by $y(x)$, integrating the result from 0 to 1 , and taking into account boundary conditions (1.2) and (1.3) we get

$$
\begin{gathered}
\int_{0}^{1}\left\{p(x) y^{\prime 2}(x)+q(x) y^{2}(x)\right\} d x+N[y]=\lambda \int_{0}^{1} y^{2}(x) d x+ \\
\int_{0}^{1} g\left(x, y(x), y^{\prime}(x), \lambda\right) y(x) d x .
\end{gathered}
$$

If $\lambda=0$, then it follows from this relation that

$$
\begin{gathered}
\int_{0}^{1}\left\{p(x) y^{\prime 2}(x)+q(x) y^{2}(x)\right\} d x+N[y]= \\
\int_{0}^{1} g\left(x, y(x), y^{\prime}(x), 0\right) y(x) d x .
\end{gathered}
$$

By virtue of (2.4) the left hand-side of (4.1) is positive, while by condition (1.5) the right hand-side of this relation is nonpositive, giving a contradiction. The proof of this lemma is complete.

Theorem 4.1. For each $n \in \mathbb{N}$, each $\sigma \in\{+,-\}$ and each $\nu \in\{+,-\}$ there exists a continuum $\hat{\mathcal{C}}_{k}^{\sigma, \nu}$ of solutions of problem (1.1)-(1.3) which contains $\left(\lambda_{n, \sigma}, \hat{0}\right)$ is contained in $\left(\mathbb{R}^{\sigma} \times \hat{S}_{n}^{\sigma, \nu}\right) \cup\left\{\left(\lambda_{n, \sigma}, \hat{0}\right)\right\}$ and is unbounded in $\mathbb{R} \times \hat{E}$.

Proof. By Remark 2.1 it follows from [15, Ch. 4, §2, Theorem 2.1] that for each $n \in \mathbb{N}$ and each $\sigma \in\{+,-\}$ the point $\left(\lambda_{n \sigma}, \hat{0}\right) \in \mathbb{R}^{\sigma} \times \hat{E}$ is a bifurcation point of problem (3.4) and a connected branch $\hat{\mathcal{C}}_{n}^{\sigma *}$ of nontrivial solutions corresponds to this point. Let $\hat{\mathcal{C}}_{n}^{\sigma}=\hat{\mathcal{C}}_{n}^{\sigma *} \cup\left\{\left(\lambda_{n \sigma}, \hat{0}\right)\right\}$. Then it follows from [17, Theorem 1.3] that either

(i) $\hat{\mathcal{C}}_{n}^{\sigma}$ is unbounded in $\mathbb{R} \times \hat{E}$, or

(ii) there exists $\left(n^{\prime}, \sigma^{\prime}\right) \neq(n, \sigma)$ such that $\left(\lambda_{n^{\prime}, \sigma^{\prime}}, \hat{0}\right) \in \hat{\mathcal{C}}_{n}^{\sigma}$.

Since $\hat{\mathcal{C}}_{n}^{\sigma}$ is connected in $\mathbb{R} \times \hat{E}$, Lemma 3.2 implies that $\hat{\mathcal{C}}_{n}^{\sigma} \subset \mathbb{R}^{\sigma} \times \hat{E}$. Next, if $(\lambda, \hat{y}) \in \hat{\mathcal{C}}_{n}^{\sigma}$ and is near $\left(\lambda_{n, \sigma}, \hat{0}\right)$, then by [17, Lemma 1.24] we have

$$
\hat{y}=\gamma \hat{y}_{n}^{\sigma+}+\hat{w},
$$

where $\hat{w}=o(|\gamma|)$ and $\hat{y}_{n}^{\sigma+}$ is an unique eigenfunction corresponding to the eigenvalue $\lambda_{n}^{\sigma}$ of (3.5) such that $\hat{y}_{n}^{\sigma+} \in \hat{S}_{n}^{\sigma,+}$ and $\left\|\hat{y}_{n}^{\sigma+}\right\|_{1}=1$. Recall that $\hat{S}_{n}^{\sigma}$ is an open set in $\mathbb{R} \times \hat{E}$, and consequently,

$$
\hat{y} \in S_{n}^{\sigma} \text { and }\left(\hat{\mathcal{C}}_{n}^{\sigma *} \cap \hat{B}_{\varepsilon}\right) \subset \mathbb{R}^{\sigma} \times \hat{S}_{n}^{\sigma}
$$

for all small $\varepsilon>0$, where $\hat{B}_{\varepsilon}=\left\{\hat{y} \in \hat{E}:\|\hat{y}\|_{1}<\varepsilon\right\}$. Moreover, it follows from Lemma 4.1 that

$$
\hat{\mathcal{C}}_{n}^{\sigma *} \cap \partial \hat{S}_{n}^{\sigma}=\emptyset,
$$


which implies that

$$
\hat{\mathcal{C}}_{n}^{\sigma} \subset\left(\mathbb{R}^{\sigma} \times \hat{S}_{n}^{\sigma}\right) \cup\left\{\left(\lambda_{n}^{\sigma}, \hat{0}\right)\right\} .
$$

Since for each $\sigma \in\{+,-\}$ the relation

$$
\hat{S}_{n}^{\sigma} \cap \hat{S}_{k}^{\sigma}=\emptyset, n, k \in \mathbb{N}, n \neq k,
$$

holds it follows that alternative (ii) of [17, Theorem 1.3] does not occur.

Now we can decompose $\hat{\mathcal{C}}_{n}^{\sigma}$ into two subcontinua $\hat{\mathcal{C}}_{n}^{\sigma,+}$ and $\hat{\mathcal{C}}_{n}^{\sigma,-}$ by using the Dancer's construction given in [11] (here $\hat{\mathcal{C}}_{n}^{\sigma \nu}=\hat{\mathcal{C}}_{n}^{\sigma *, \nu} \cup\left\{\left(\lambda_{n}^{\sigma}, \hat{0}\right)\right\}$ ). It is obvious that $t \hat{y}_{n}^{\sigma+} \in S_{n}^{\sigma, \pm}$ for $\pm t>0$. Consequently, if $(\lambda, \hat{y}) \in \hat{\mathcal{C}}_{n}^{\sigma+}\left(\hat{\mathcal{C}}_{n}^{\sigma-}\right)$ and is near $\left(\lambda_{n}^{\sigma}, \hat{0}\right)$, then by $(4.2)$ we have

$$
\left(\hat{\mathcal{C}}_{n}^{\sigma *,+} \cap \hat{B}_{\varepsilon}\right) \subset \mathbb{R}^{\sigma} \times \hat{S}_{n}^{\sigma,+}\left(\left(\hat{\mathcal{C}}_{n}^{\sigma *,-} \cap \hat{B}_{\varepsilon}\right) \subset \mathbb{R}^{\sigma} \times \hat{S}_{n}^{\sigma,-}\right)
$$

for all small $\varepsilon>0$. Moreover, in view of Lemma 4.1 we get

$$
\hat{\mathcal{C}}_{n}^{\sigma *,+} \cup \partial \hat{S}_{n}^{\sigma,+}=\emptyset \text { and } \hat{\mathcal{C}}_{n}^{\sigma *,-} \cup \partial \hat{S}_{n}^{\sigma,-}=\emptyset .
$$

Therefore, we have the following relations

$$
\hat{\mathcal{C}}_{n}^{\sigma,+} \subset\left(\mathbb{R}^{\sigma} \times \hat{S}_{n}^{\sigma,+}\right) \cup\left\{\left(\lambda_{n}^{\sigma}, \hat{0}\right)\right\} \text { and } \hat{\mathcal{C}}_{n}^{\sigma,-} \subset\left(\mathbb{R}^{\sigma} \times \hat{S}_{n}^{\sigma,-}\right) \cup\left\{\left(\lambda_{n}^{\sigma}, \hat{0}\right)\right\} .
$$

Since

$$
\hat{S}_{n}^{\sigma,+} \cap \hat{S}_{n}^{\sigma,-}=\emptyset, n \in \mathbb{N}
$$

it follows that

$$
\hat{\mathcal{C}}_{n}^{\sigma *,+} \cap \hat{\mathcal{C}}_{n}^{\sigma *,-}=\emptyset, n \in \mathbb{N} .
$$

Then by virtue of Theorem 2 of [9] the sets $\hat{\mathcal{C}}_{n}^{\sigma *,+}$ and $\hat{\mathcal{C}}_{n}^{\sigma *,-}$ are unbounded in $\mathbb{R} \times \hat{E}$. The proof of this theorem is complete.

By (4.2) from Theorem 3.1 we have the following result.

Theorem 4.2. For each $n \in \mathbb{N}$, each $\sigma \in\{+,-\}$ and each $\nu \in\{+,-\}$ there exists a continuum $\mathcal{C}_{k}^{\sigma, \nu}$ of solutions of problem (1.1)-(1.3) which contains $\left(\lambda_{n, \sigma}, 0\right)$ is contained in $\left(\mathbb{R}^{\sigma} \times S_{n}^{\sigma, \nu}\right) \cup\left\{\left(\lambda_{n, \sigma}, 0\right)\right\}$ and is unbounded in $\mathbb{R} \times E$.

Now suppose that the nonlinear term $g$ has the form $g(x, u, s, \lambda)=g_{1}(x, u, s, \lambda) u$ where $g_{1} \in C^{0}\left([0,1] \times \mathbb{R}^{3}\right)$ and satisfies the following conditions:

$$
g_{1}(x, u, s, \lambda) \leq 0,(x, u, s, \lambda) \in[0,1] \times \mathbb{R}^{3} ;
$$

there is a constant $K>0$ such that

$$
\left|g_{1}(x, u, s, \lambda)\right| \leq K, \quad(x, u, s, \lambda) \in[0,1] \times \mathbb{R}^{3} .
$$

Theorem 4.3. Let the conditions (4.3) and (4.4) be satisfied. Then

$$
\mathcal{C}_{n}^{+, \nu} \subset\left(I_{n}^{+} \times S_{n}^{+, \nu}\right) \cup\left\{\left(\lambda_{n}^{+}, \hat{0}\right)\right\} \text { and } \mathcal{C}_{n}^{-, \nu} \subset\left(I_{n}^{-} \times S_{n}^{-, \nu}\right) \cup\left\{\left(\lambda_{n}^{-}, \hat{0}\right)\right\}
$$

for each $n \in \mathbb{N}$ and each $\nu \in\{+,-\}$, where

$$
I_{n}^{+}=\left[\lambda_{n}^{+}, \lambda_{n,+}^{K}\right], I_{n}^{-}=\left[\lambda_{n,-}^{K}, \lambda_{n}^{-}\right],
$$

and $\lambda_{n,+}^{K}$ and $\lambda_{n,-}^{K}$ are $n$-th positive and negative eigenvalues of problem

$$
\begin{aligned}
& \left(p(x) y^{\prime}(x)\right)^{\prime}+(q(x)+K) y(x)=\lambda r(x) y(x), x \in(0,1), \\
& b_{0} y(0)=d_{0} p(0) y^{\prime}(0),\left(a_{1} \lambda+b_{1}\right) y(1)=p(1) y^{\prime}(1),
\end{aligned}
$$

respectively. 
Proof. We will prove the theorem for arbitrary fixed $n=n_{0}, \nu=\nu_{0}$, and $\sigma=+$ (the case of $\sigma=-$ is considered in a similar way).

Let $(\tilde{\lambda}, \tilde{y})$ be a solution of problem (1.1)-(1.3). Then $(\tilde{\lambda}, \tilde{y})$ solves the following linear eigenvalue problem

$$
\begin{aligned}
& \left(p(x) y^{\prime}(x)\right)^{\prime}+(q(x)+\tilde{h}(x)) y(x)=\lambda r(x) y(x), x \in(0,1), \\
& b_{0} y(0)=d_{0} p(0) y^{\prime}(0),\left(a_{1} \lambda+b_{1}\right) y(1)=p(1) y^{\prime}(1),
\end{aligned}
$$

where

$$
\tilde{h}(x)=-g_{1}\left(x, \tilde{y}(x), \tilde{y}^{\prime}(x), \tilde{\lambda}\right) .
$$

It follows from conditions (4.3) and (4.4) that

$$
\tilde{h}(x) \in C^{0}[0,1] \text { and } 0 \leq \tilde{h}(x) \leq K \text { for } x \in[0,1] .
$$

By (2.7), (2.9) and (4.7) it follows from [16, Lemma 2.2] that

$$
\lambda_{n,+} \leq \lambda_{n,+}^{\tilde{h}} \leq \lambda_{n,+}^{K}, n \in \mathbb{N},
$$

where $\lambda_{n,+}^{\tilde{h}}$ is the $n$-th positive eigenvalue of problem (4.6).

Thus, if $(\tilde{\lambda}, \tilde{y}) \in \mathcal{C}_{n_{0}}^{+*, \nu_{0}}$, then $\tilde{\lambda}=\lambda_{n,+}^{\tilde{h}}$, and consequently, $\tilde{\lambda} \in I_{n_{0}}^{+}$in view of (4.8). The proof of this theorem is complete.

\section{References}

[1] Z.S. Aliev, On the global bifurcation of solutions of some nonlinear eigenvalue problems for ordinary differential equations of fourth order, Sb. Math. 207 (2016), no. 12, 3-29.

[2] Z.S. Aliyev, G.M. Mamedova, Some global results for nonlinear Sturm-Liouville problems with spectral parameter in the boundary condition, Ann. Polon. Math. 115 (2015), no. 1, 75-87.

[3] Z.S. Aliyev, L.V. Nasirova (Ashurova), Bifurcation of positive and negative solutions of nonlinearizable Sturm-Liouville problems with indefinite weight, Miskolc Math. Notes 21 (2020), no. 1, 19-29.

[4] L.V. Ashurova, Global bifurcation of solutions for the problem of population modeling, Caspian J. Appl. Math., Ecol. and Econom. 5 (2017), no. 1, 65-71.

[5] H. Berestycki, On some nonlinear Sturm-Liouville problems, J. Diffential Equations 26 (1977), 375-390.

[6] P.A. Binding, P.J. Browne, B.A.Watson, Spectral problem for nonlinear SturmLiouville equations with eigenparameter dependent boundary conditions, Canad. J. Math. 52 (2000), no. 2, 248-264.

[7] P.A. Binding, P.J. Browne, B.A.Watson, Left definite Sturm-Liouville problems with eigenparameter dependent boundary conditions, Differential Integral Equations 12 (1999), 167-182.

[8] G. Dai, R. Ma, Bifurcation from intervals for Sturm-Liouville problems and its applications, Electron. J. Differ. Equ. 2014, no. 03, 10 pp.

[9] E. N. Dancer, On the structure of solutions of non-linear eigenvalue problems, Indiana Univ. Math. J. 23 (1974), 1069-1076.

[10] R.S. Cantrell, C. Cosner, Spatial Ecology via Reaction-Diffusion Equations, Ser. Math. Comput. Biol., John Wiley and Sons Ltd., Chichester, UK (2003).

[11] R. Courant and D. Hilbert, Methoden der Mathematischen Physik, I, Springer, Berlin, 1931; English transl., Interscience, New York, 1953.

[12] W.H. Fleming, A selection-migration model in population genetics. J. Math. Biology 2 (1975), 219-233. 
[13] C.T. Fulton, Two-point boundary value problems with eigenvalue parameter contained in the boundary conditions, Proc. Roy. Soc. Edinburgh Sect. A 77 (1977), no. 3-4, 293-308

[14] P. Hess, T. Kato, On some linear and nonlinear eigenvalue problems with indefinite weight function, Comm. Partial Differential Equations 5 (1980), 999-1030.

[15] M. A. Krasnoselski, Topological methods in the theory of nonlinear integral equations, Macmillan, New York, 1965.

[16] L.V. Nasirova, Global bifurcation from intervals of solutions of nonlinear SturmLiouville problems with indefinite weight, Trans. Natl. Acad. Sci. Azerb. Ser. Phys.Tech. Math. Sci. Mathematics 39 (2019), no. 4, 148-154

[17] P.H. Rabinowitz, Some global results for nonlinear eigenvalue problems, J. Funct. Anal. 7 (1971), 487-513.

[18] B.P. Rynne, Bifurcation from zero or infinity in Sturm-Liouville problems which are not linearizable, J. Math. Anal. Appl. 228 (1998), 141-156.

[19] K. Schmitt, H. L. Smith, On eigenvalue problems for nondifferentiable mappings, $J$. Differential Equations 33 (1979), no. 3, 294-319.

[20] J. Walter, Regular eigenvalue problems with eigenvalue parameter in the boundary conditions. Math. Z. 133 (1973), 301-312.

Ulkar V. Gurbanova

Ganja State University, Ganja AZ 2000, Azerbaijan

E-mail address: ulya1812dok2@mail.ru

Received: May 19, 2020; Revised: November 28, 2020; Accepted: December 14, 2020 